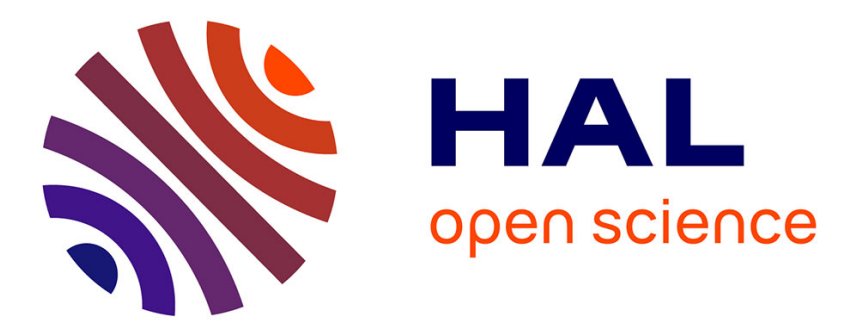

\title{
Spreading of non volatile liquids on smooth solid surfaces : role of long range forces
}

L. Léger, M. Erman, A.M. Guinet-Picart, D. Ausserre, C. Strazielle, J.J. Benattar, F. Rieutord, J. Daillant, Louis Bosio

\section{- To cite this version:}

L. Léger, M. Erman, A.M. Guinet-Picart, D. Ausserre, C. Strazielle, et al.. Spreading of non volatile liquids on smooth solid surfaces: role of long range forces. Revue de Physique Appliquée, 1988, 23 (6), pp.1047-1054. 10.1051/rphysap:019880023060104700 . jpa-00245907

\section{HAL Id: jpa-00245907 https://hal.science/jpa-00245907}

Submitted on 1 Jan 1988

HAL is a multi-disciplinary open access archive for the deposit and dissemination of scientific research documents, whether they are published or not. The documents may come from teaching and research institutions in France or abroad, or from public or private research centers.
L'archive ouverte pluridisciplinaire HAL, est destinée au dépôt et à la diffusion de documents scientifiques de niveau recherche, publiés ou non, émanant des établissements d'enseignement et de recherche français ou étrangers, des laboratoires publics ou privés. 
Classification

Physics Abstracts

$68.10 \mathrm{G}-61.25 \mathrm{H}$

\title{
Spreading of non volatile liquids on smooth solid surfaces : role of long range forces
}

\author{
L. Léger, M. Erman ( $\left.{ }^{1}\right)$, A. M. Guinet-Picart, D. Ausserre (**), C. Strazielle $\left({ }^{2}\right)$, \\ J. J. Benattar $\left({ }^{3}\right)$, F. Rieutord ( $\left.{ }^{3}\right)$, J. Daillant $\left({ }^{3}\right)$ and L. Bosio ( $\left.{ }^{4}\right)$ \\ Laboratoire de Physique de la Matière Condensée, Collège de France, Associated with C.N.R.S., UA 792, \\ 75231 Paris Cedex 05, France \\ (1) Laboratoire d'Electronique et de Physique Appliquée, 3 Avenue Descartes, 94451 Limeil-Brévannes \\ Cedex, France $(*)$ \\ (2) Institut Charles Sadron, C.N.R.S., 6 rue Boussingault, 67083 Strasbourg, France \\ (3) Department PhG/SPRSRM, C.E.N. Saclay, 91190 Gif-sur-Yvette, France \\ (4) ESPCI, 10 rue Vauquelin, 75231 Paris Cedex 05, France
}

(Reçu le 4 novembre 1987, révisé le 29 janvier 1988, accepté le 4 février 1988)

\begin{abstract}
Résumé. - Nous présentons une étude expérimentale de l'étalement de gouttes de polydiméthyl siloxane (liquide non volatile) sur la surface de pastilles de silicium (surface lisse, chimiquement bien contrôlée). La partie macroscopique de la goutte (qui peut être observée à l'œil nu ou au microscope) a un comportement nettement distinct de celui de la partie microscopique, ou film précurseur, qui se développe lentement comme une fine langue de liquide (épaisseur plus petite que $1000 \AA$ ) tout autour de la goutte. L'étalement de la goutte macroscopique ne dépend pas du paramètre d'étalement tandis que le film précurseur est profondément influencé par les énergies de surface. Nous avons caractérisé ce film précurseur (profil et cinétiques d'évolution) par des techniques complémentaires telles que l'observation en lumière réfléchie polarisée sous microscope, l'ellipsométrie résolue spatialement et la réflectivité des rayons $\mathrm{X}$. Les résultats sont comparés à des modèles théoriques récents et montrent des effets spécifiquement polymères.
\end{abstract}

\begin{abstract}
We present an experimental investigation of the spreading of non volatile polydimethylsiloxane drops on smooth horizontal silicone wafers. We distinguish the macroscopic part of the drop which can be seen by bare eye (or through a microscope) from the microscopic part, or precursor film, which progressively extends like a thin liquid tongue (thickness smaller than $1000 \AA$ ) all around the drop. The macroscopic drop spreads independently of the spreading parameter, in opposition to the precursor film which appears deeply influenced by the surface energies. We have characterized this precursor film (both its profile and its time evolution) by complementary techniques such as ellipsocontrast microscopic observations, spatially resolved ellipsometry, X-rays reflectivity. The results will be compared with recent theoretical predictions, and specific polymeric effects will be emphasized.
\end{abstract}

\section{Introduction.}

The spreading of a liquid on a solid surface is of obvious importance in a number of practical situations (paints, textile dying, metal or glasses anticorrosive coaling, lubrication, gluing, plants treatments, ...). The underlying mechanisms are, however, only

(*) LEPA : Member of the Philips Research Organization.

(**) Present address : IBM Research, Almaden Research Center, 650 Harry Road, San Jose, California 95120-6099, U.S.A. poorely understood. Recent theoretical approaches have been developed by Scriven et al. [1], de Gennes [2] and Joanny [3], which emphasize the role of long range cohesive forces in the liquid on the spreading process. Following these approaches, the behaviour of volatile and non volatile liquids are clearly different : a volatile liquid is always in equilibrium with its vapour; molecules of the liquid can be efficiently transported through the vapour phase very far from the liquid front. Thus, when a drop of a volatile liquid is deposited on a solid, in a situation in which the liquid wets the solid, the surface is very rapidly covered by a thin layer of recondensed liquid 
molecules coming from the vapour. On the contrary, if the liquid has a very low vapour tension, the only efficient way of covering the solid surface by a liquid film is by flow of the liquid. The kinetics of the process, due to viscous dissipation in the thin liquid film formed, will be strongly slowed down compared to the volatile case. Moreover, not only the kinetics, but also the final equilibrium state of spreading will be quite different in the two situations. One can guess that the final state of spreading is a thin flat liquid layer. If the thickness of the liquid film becomes smaller than the range of the cohesive interactions in the liquid, the two interfaces solidliquid and liquid-gas become correlated: if one wants to make the liquid still thinner, one has to break cohesive interactions on both sides, and the energy of the thin film is larger than that of a film having a thickness larger than the range of the interactions. A way of taking this effect into account is to introduce a pressure, the disjoining pressure $\pi(z)$ [4], which increases when $z$ goes to zero. For a volatile liquid, the final state of the spreading film corresponds to an equilibrium between the molecules in the film (at the pressure $\pi(z))$ and in the vapour phase. The equilibrium thickness of the film is thus fixed by an external parameter, the degree of saturation of the vapour. On the contrary, when the liquid is non volatile, the total volume of the liquid is fixed; make the film thinner means that a larger area of the solid surface will be covered, and the film thickness is the result of a balance between two antagonist effects : disjoining pressure which tends to thicker the film and spreading forces which tend to make it thinner.

The spreading of a non volatile liquid thus appears to be more directly linked to the intrinsic properties of the solid/liquid system, and a detailed description of the process has been proposed by Joanny and de Gennes [2, 3]. It is tempting to compare these predictions with the large number of available experimental work performed on the subject, owing to its practical importance [5]. However, many of these experiments have been performed on volatile liquids or, when silicone oils were used, on not well controlled surfaces. For this reason, we have undertaken systematic spreading experiments on a model system (polydimethyl siloxane (PDMS) deposited on smooth silicon wafers), using complementary techniques, in order to characterize the drop behaviour at all length scales. In part 1 we shall present the experimental system and the different techniques used. In part 2 the present results will be gathered both for large drops where macroscopic and microscopic (precursor film) regimes coexist, even at very long spreading times, and for tiny drops which are rapidly thin enough to be entirely affected by long range forces. In part 3, these results will be discussed and compared with theoretical predictions.

\section{Experimental system and techniques.}

To ensure that the liquid we used was not volatile, we have chosen a polymer, the polydimethylsiloxane. It is a flexible chain, well in the liquid phase at room temperature (its glass transition temperature is $T_{\mathrm{g}}=-120^{\circ} \mathrm{C}$ ). The choice of a polymer is convenient, as the viscosity can be varied in a wide range by varying the molecular weight ( $\eta \sim M^{3.4}$ for well entangled systems [6]), without affecting the interactions. However, polymers are always polydisperse, especially silicon oils which quite often contain a large amount of low molecular weight oligomers which are volatile and thus may strongly perturbate the results. To avoid this difficulty (or limit it as much as we could) we have used narrow fractions obtained (by fractionated precipitation) and characterized by one of us (C.S.) in Strasbourg. The characteristics of the polymer samples used in the present study are listed in table I.

Table I.

\begin{tabular}{|c|c|c|}
\hline$M_{w}$ & $M_{w} / M_{n}$ & $\eta$ (Poises) \\
\hline 6500 & 2 & 0.5 \\
\hline 79000 & 1.15 & 350 \\
\hline 160000 & 1.11 & 4260 \\
\hline 280000 & 1.19 & 24500 \\
\hline
\end{tabular}

The surface tension of the polymer is $\gamma \sim 21-22 \mathrm{mN} / \mathrm{m}$. The solid surface, silicon wafer, has been chosen because of its high reproducible chemical quality, and of its very small residual roughness. A silicon wafer is a slab of a silicon monocrystal, cut in our case along a [III] plane, covered by a thin ( $20 \AA$ thick) oxide layer. In order to test the influence of the spreading power of the surface, we have achieved two surface states : a high energy surface obtained by strong oxidation of the organic surface impurities by oxygen flow under UV irradiation [7] an efficient cleaning procedure which does not affect the surface roughness, and a low energy surface obtained by chemical grafting of a compact monolayer of octadecyltrichlorosilane, following Sagiv's procedure [8]. The spreading power of the surface can be quantified by the spreading parameter $S=\gamma_{\mathrm{SG}}-\gamma_{\mathrm{SL}}-\gamma$ with $\gamma_{\mathrm{SL}}$ and $\gamma$ the solid-gas, solid-liquid, liquid-gas interfacial tensions respectively. $S$ represents the energy gained by covering one unit area of the solid by a thick liquid film. In the first case (clean wafer) water spreads on the surface, which has thus a critical surface tension $\gamma_{\mathrm{C}}$ [9] larger than $75 \mathrm{mN} / \mathrm{m}$ leading to large $S$ values 
(an estimation of $S$ is $S=\gamma_{\mathrm{C}}-\gamma \geq 50 \mathrm{mN} / \mathrm{m}$ ). On the contrary $\gamma_{\mathrm{C}}$, deduced from contact angle measurements of alcanes on the grafted surface, is of the order of 22 to $23 \mathrm{mN} / \mathrm{m}$ and $S$ is smaller or close to 1 , but still positive (the liquid still spreads).

A typical experiment is performed in the following way: a small drop (volume $\Omega$ varying between $10^{-7}$ and $10^{-4} \mathrm{~cm}^{3}$ ) is deposited on the wafer which is immediately enclosed in a sealed box equipped with a glass window in order to prevent further contamination by dust or atmosphere impurities during the spreading period which may last for several months. Up to now, the atmosphere around the drop has not been further controlled (no special care was taken to avoid humidity, or work under inert nitrogene atmosphere). Then the size $R$ of the macroscopic drop and its apparent contact angle $\theta_{\mathrm{a}}$ are measured periodically by direct observation through a microscope (size and small contact angle measurements through equal thickness fringe spacing) or using a recently proposed method in which the drop is used as a convex mirror, the cone inside which the light is reflected having an angle 2 $\theta_{\mathrm{a}}$ [10]. At the same time, a careful examination of the system, through a microscope in polarized re- flected light, reveals a bright halo all around the macroscopic drop (Fig. 1). As time goes, this halo extends further and further from the visible edge of the drop (last black interference fringe) and at the same time becomes fainter. We have attributed this special contrast to the presence of a thin liquid film on the top of the silicon wafer surface. At the wavelength used (green or white light), the silicon acts as a metallic mirror. It is covered by the $20 \AA$ thick dielectric oxide layer plus eventually a liquid film. The optical properties of such a layered thin film are classical and can be calculated using the Fresnel formula [11]. The important point is that the reflection coefficient is different for a polarization in or normal to the plane of incidence $\left(r_{\|}\right.$and $r_{\perp}$ respectively), a property classically used in ellipsometry to gain information on the dielectric layer. In the present observation through a microscope, the direction of polarization of the incident light is fixed with respect to the sample, but all beams contained inside the cone of aperture of the objective are accepted to form the image. To calculate how the collected intensity is affected by the liquid film, one has to perform a double integration on all angles of incidence contained in that cone and, at fixed

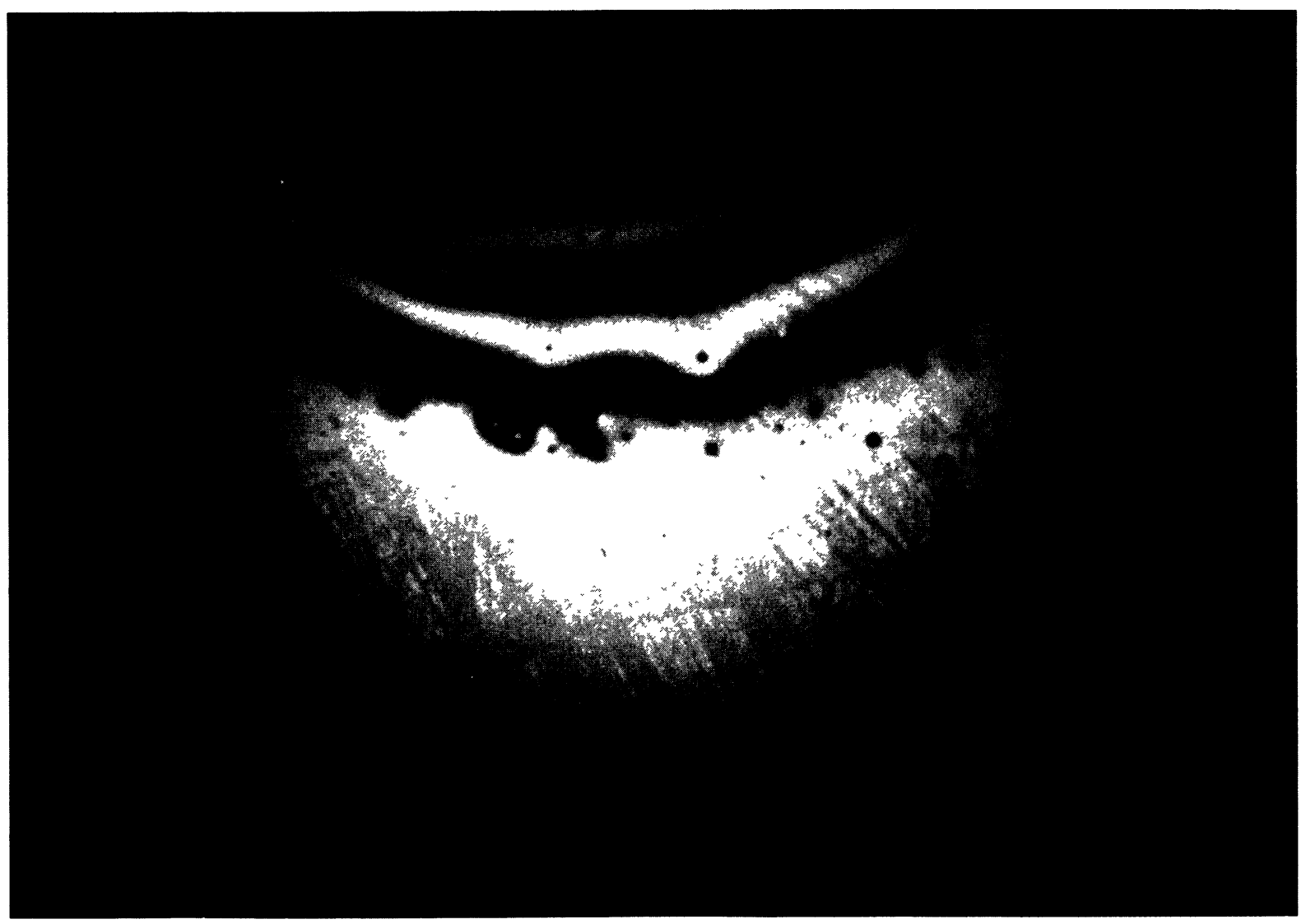

Fig. 1. - Aspect of a drop, as observed trough a microscope, by ellipsocontrast techniques. The precursor film appears like a bright halo all around the macroscopic drop. This halo is uniform when the drop is deposited on a bare wafer, but when a silanated wafer is used like in the picture, irregularities of the spreading power of the surface are revealed by the presence of the film, and attributed to inhomogeneities in the grafted monolayer. 
angle of incidence, on all possible orientations of the plane of incidence with respect to the direction of polarization. The resulting contrast thus calculated, for the optical parameters of our system is reported in figure 2 as a function of the PDMS thickness.

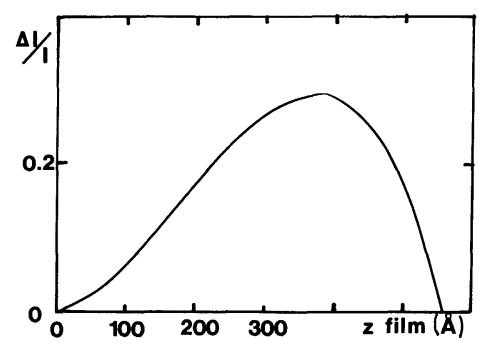

Fig. 2. - Evaluation of the contrast introduced by the film when observed in polarized reflected light, through a microscope, as a function of the film thickness. The oxide layer is assumed to be $20 \AA$ thick. The postulated optical constants of the system are : $\mathrm{Si}: n=4-i \times 0.03 \mathrm{SiO}_{2}$ : $n=1.45 ; \quad$ PDMS : $n=1.43$, and the wavelength $\lambda=0.5 \mu \mathrm{m}$. The incident angle is $20^{\circ}$.

Observation in polarized reflected light, through a microscope, reveals itself a useful tool to study inhomogeneous thin films, a method that we have called ellipsocontrast [12]. It appears quite sensitive for thicknesses in the range $100 \AA-500 \AA$ (for the optical constants of our system), and has the strong advantage of a high spatial resolution $(1 \mu \mathrm{m})$. The sensitivity rapidly decreases for smaller thicknesses, in opposition to conventional ellipsometry, because the aperture of the microscope is not large enough to allow for incidence angles close to Brewster's angle.

In order to extend the range of thicknesses investigated, we have also used ellipsometry, with a high performance ellipsometer, built by one of us (M. E.) and Theeten, in the Laboratoire d'Electronique et de Physique Appliquée (LEPA), and particularly designed to have a good spatial resolution [13]. The size of the illuminated area on the sample is $10 \times 25 \mu \mathrm{m}$. A light beam of fixed polarization and fixed incidence angle is shined on the sample. The reflected intensity is collected through a rotating analyser, and the two ellipsometric parameters $\cos \Delta$ and $\operatorname{tg} \psi$, with $\frac{\eta_{I}}{r_{\perp}}=\operatorname{tg} \psi \mathrm{e}^{i \Delta}$, extracted from the amplitude and phase of the component of the reflected intensity modulated at twice the rotation frequency of the analyser [13]. From $\operatorname{tg} \psi$ and $\cos \Delta$, the index of refraction and the thickness of a uniform dielectric layer can classically be extracted [14]. In the present experiments, we have assumed the thickness locally uniform in the illuminated window, treated the oxide layer (index of refraction $n=1.45)$ and the PDMS $(n=1.43)$ as the same optical medium, and extracted the film thickness $z$ as a function of the position through the drop from the $\cos \Delta, \operatorname{tg} \psi$ data. Such an inversion of ellipsometric data, even if classical, is not totally obvious, and the procedure used - with its limitations - is discussed in details in reference [15]. Typically, thicknesses from 0 to $1000 \AA$ can be determined with an accuracy of a few $\AA$ [13].

The last technique used to characterize the wafer surface and the final states of spreading is X-rays reflectometry [16]. The experiments have been performed in Saclay and ESPCI by J. J. B., F. R., J. D. and L. B. The spatial resolution is limited $(1 \mathrm{~mm} \times 0.7 \mathrm{~mm})$. For X-rays, the sample is again a layered structure with an electronic density different for silicon, silica oxide layer, aliphatic chains grafted layer, and PDMS. If the electronic densities and thicknesses are known, the reflectivity $R$ can be calculated as a function of the angle of incidence, $\theta$, from the Fresnel formula. In a reverse way, the shape of the curve $R(\theta)$ can be fitted to extract the parameters of the layers. Departures from the Fresnel curve give estimations of the roughness at angles not too far from the critical angle. At large angles, interferences on the layered structure appear and allow to extract the layer thickness with an accuracy of the order of the $\AA$. For the bare wafers, the reflectivity curves yield a surface roughness of $4.5 \AA$. For the grafted layer they reveal neat variations in the grafted layer characteristics from one grafting experiment to another. In the best cases a completely dense layer is obtained, with a thickness equal to the full-length of the grafted chain normal to the surface. More often irregularities are observed and we interpret them as dense zone separated by less dense regions in which the chains are slightly tilted. On the totally dense grafted layer, PDMS no longer spreads $(S \leq 0)$. Inhomogeneities in the structure of the grafted layer correspond to inhomogeneities in the $S$ values.

\section{Results.}

2.1 MACROSCOPIC SPREADING KINETICS. - The time evolution of the size and of the apparent contact angle on bare and grafted wafers has yet been reported [12]. We just recall here the main results which are illustrated by figure 3 . All the drops studied are well inside the capillary regime $\left(R<K^{-1}\right.$ with the capillary length

$$
K^{-1}=\left(\frac{2 \gamma}{\rho_{g}}\right)^{\frac{1}{2}} \sim 2.2 \mathrm{~mm}
$$

for our system). Both, the size and the apparent contact angle follow universal scaling laws with time :

$$
\begin{aligned}
& R(t) \sim t^{0.1 \pm 0.01} \\
& \theta_{\mathrm{a}}(t) \sim t^{-0.3 \pm 0.01}
\end{aligned}
$$




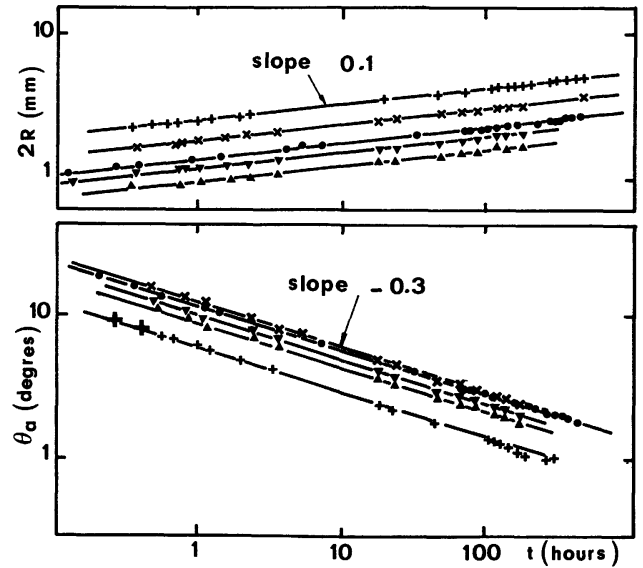

Fig. 3. - Spreading kinetics of the macroscopic part of the drops, characterized by their size $R$ and their apparent contact angle $\theta_{\mathrm{a}}$, for a wide range of drop characteristics, and two spreading parameter values : $(\bullet)$ Clean wafer, $M_{w}=1.6 \times 10^{5}, \quad \Omega=6.5 \times 10^{-5} \mathrm{~cm}^{3} ; \quad(\times)$ Silanated wafer, $M_{w}=1.6 \times 10^{5}, \Omega=1.8 \times 10^{-4} \mathrm{~cm}^{3} ;(\nabla)$ Silanated wafer, $M_{w}=1.6 \times 10^{5}, \quad \Omega=3.5 \times 10^{-5} \mathrm{~cm}^{3}$; $(\triangle)$ Silanated wafer, $M_{w}=1.6 \times 10^{5}, \quad \Omega=1.5 \times$ $10^{-5} \mathrm{~cm}^{3}$; (+) Silanated wafer, $M_{w}=7.9 \times 10^{4}, \Omega=$ $2.1 \times 10^{-4} \mathrm{~cm}^{3}$.

The exponents are independent of the spreading parameter, and in very good agreement with Tanner's laws [17].

For very small drops, an acceleration of the spreading process is observed $\left(R(t) \sim t^{\alpha}\right.$ with $\alpha>0.1$, but $R^{3} \theta_{\text {a }}$ remains a constant.

2.2 PRECURSOR FILM CHARACTERIZATIONS. - Figure 1 is a visualization by ellipsocontrast of a precursor film developed on a grafted wafer. Strong irregularities of the film thickness are immediately visible, and revealed by the presence of the film (they were totally invisible before the film deposition, even using sensitivie Normarski's techniques).

In figure 4 an analogous illustration is obtained through a cartography performed by ellipsometry. The lines in figure $4 \mathrm{~b}$ are equal $\cos \Delta$ lines, i.e. equal thickness lines. Again strong irregularities are clearly visible. These irregularities are not present when a bare wafer is used. For this reason, a detailed quantitative analysis of precursor film profiles has only been conducted, at the present time, on bare wafers.

In figure 5 two precursor film profiles obtained on very different drops (characteristics detailed in the figure caption) are reported. The profiles are cut at the transition towards the macroscopic drop, a point which can be precisely located, as the slope of the free surface there becomes large enough to prevent the ellipsometric inversion (assuming a locally uniform film) to be valid. We can notice in figure 5 that the precursor film appears much more developed
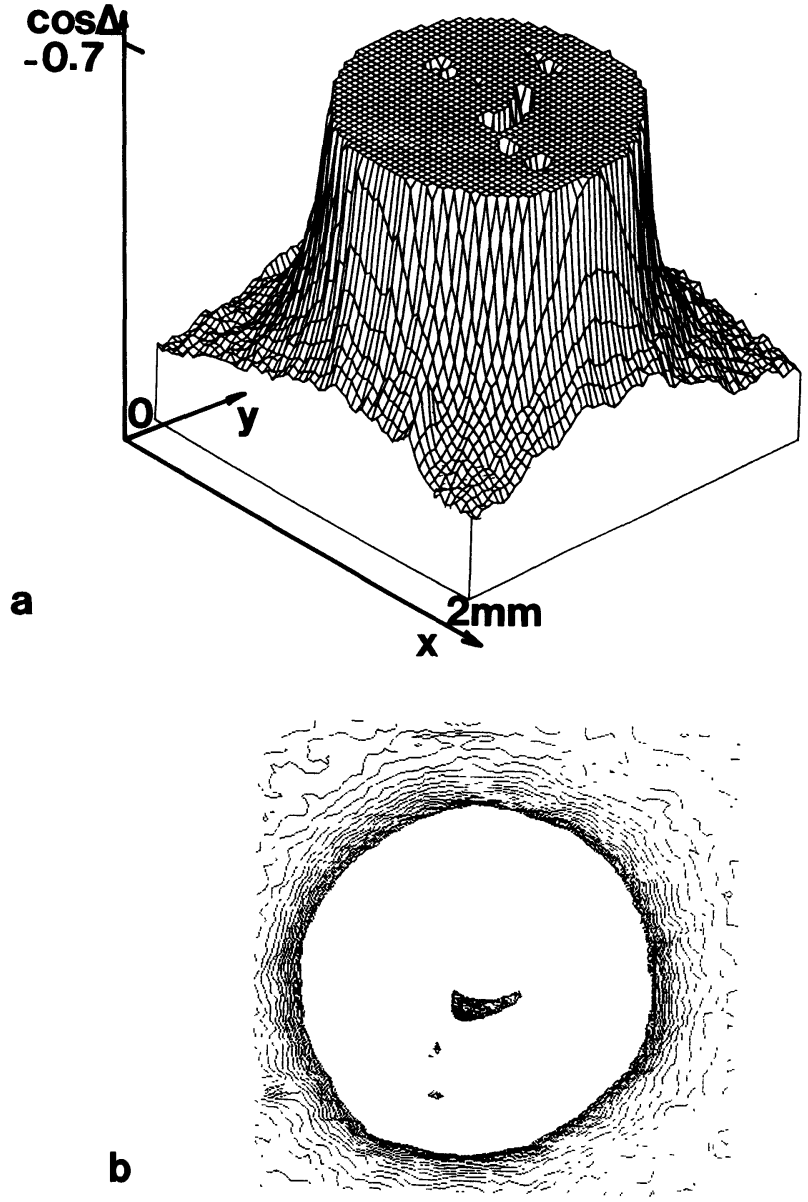

Fig. 4. - Ellipsometric cartography of the thin precursor film zone, for a drop deposited on a grafted wafer : (a) $\cos \Delta$ as a function of the position $(x, y)$ in the wafer plane ; (b) Equal cos $\Delta$ lines (i.e. equal thickness lines), displaying an overall axial symetry, plus strong fluctuations, related to the inhomogeneities of the grafted silane monolayer. For a better visualization, the cartography is truncated at $\cos \Delta=-0.7$, i.e. thicknesses of the order of $250 \AA$.

(thickness and extension) for the drop having the smaller apparent contact angle. No extremity of the film can be located on these profiles.

In figure 6 , a rather young precursor film profile for a relatively high molecular weight PDMS is reported. It shows a non monotonic decrease of thickness. This bump, which appears for thicknesses comparable to the radius of gyration of the chains, slowly disappears when the film evolves and develops.

2.3 VERY SMALL DROPS. - An ellipsometric profile of a very small drop is reported in figure 7 . The maximum thickness is of the order of $200 \AA$, which means that long range forces are important everywhere in this drop, and no macroscopic regime remains. Such drops are totally invisible by bare eye. As time goes they progressively flaten down. 


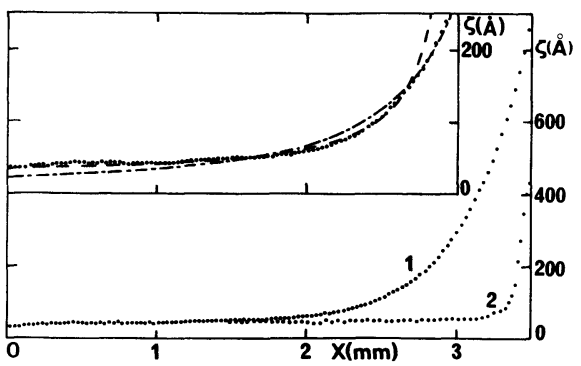

Fig. 5. - Precursor film profiles, obtained by spatially resolved ellipsometry for two drops of very different characteristics: profile number 1: molecular weight 6500 , drop volume $\Omega=1.34 \times 10^{-5} \mathrm{~cm}^{3}$, apparent contact angle $\theta_{\mathrm{a}}=0.055^{\circ}$; profile number 2 : molecular weight 280000 , volume $\Omega=6.11 \times 10^{-7} \mathrm{~cm}^{3}$, apparent contact angle $\theta_{\mathrm{a}}=0.914^{\circ}$. The two profiles have been interrupted at the transition towards the macroscopic drop. The reported thickness is that of the precursor film plus that of the oxide layer covering the wafer. The saturation, for $x \rightarrow 0$, towards $30 \AA$ corresponds to the bare wafer, i.e. to a zero film thickness. In the insert (same $x$ scale, doubled vertical scale), the dotted line is the best least square fit of the tail of profile 1 ( 80 last data points) on a curve $z \simeq 1 / x-x_{0}$.

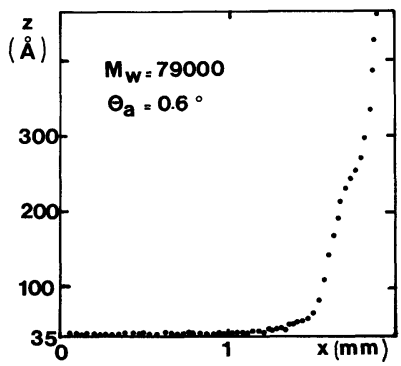

Fig. 6. - Precursor film profile, obtained by ellipsometry, of a PDMS drop of molecular weight 79000 . When the film is rather young, a bump appears for a thickness comparable to the radius of gyration of the polymer chains.

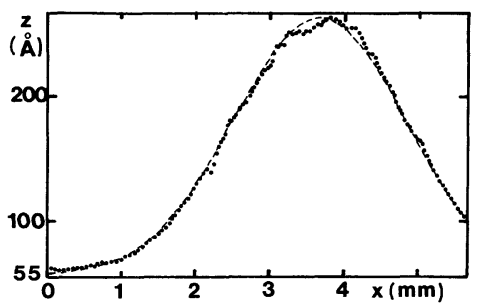

Fig. 7. - Ellipsometric profile of a very small drop. After a few days of spreading, the thickness of a drop of volume of order $5 \times 10^{-7} \mathrm{~cm}^{3}$ and of molecular weight 6500 , is small enough for producing a drop entirely sensitive to long range forces. The macroscopic drop has disappeared. The profile of such a small drop is well described by a Gaussian (dotted line).
The same drop has been followed by X-rays reflectivity. It progressively disappears, only affecting the roughness of the substrate which falls down to zero in the area covered by the film while its remains $4.5 \AA$ close to the edges of the wafer not reached by the liquid.

Such a result is slightly surprising as the size of the PDMS monomer is close to $5 \AA$. We think that the disappearance of the film, and the fact that we cannot locate the film extremity in the ellipsometric profiles has to be related with the roughness of the wafer, not negligible in front of such a small film thickness : the PDMS liquid fills the hollows of the surface, and the remaining roughness becomes almost zero.

Identical small drops deposited on a grafted wafer seem to stabilize at much larger thickness $(\sim 14 \AA)$, a very preliminary result which has to be confirmed, but may indicate that the final state of spreading is indeed conditioned by the spreading parameter.

\section{Discussion.}

All the results presented above seem to agree quite well, at least qualitatively, with de Gennes, Joanny's predictions : small spreading drops of a non volatile liquid indeed separate into two parts having very different characteristics. The macroscopic part evolves independently of the spreading power of the surface. On the contrary, the thin precursor film which slowly develops ahead the macroscopic drop appears deeply influenced by the value of the spreading parameter, as illustrated by figures 1 and 4. Even if very preliminary, the X-rays reflectivity results on the final state of spreading, seem to show that the picture of a pancake with a thickness strongly dependent on $S$ is not very different from the reality. Moreover, as for the large $S$ value the thickness of the pancake is only of a few $\AA$ (smaller than the roughness $4.5 \AA$ ), for $S$ close to 1 , and for Van der Waals interactions, one expects $[2,3]$ a thickness $e \sim a \sqrt{\frac{3 \gamma}{2 S}}$, i.e. $e \sim 5 e_{\text {bare wafer }}$ and the first measurement of $14 \AA$ has the correct order of magnitude ( $a$ has the order of magnitude of a molecular size, and we identify it with $\left.e_{\text {bare layer }}\right)$.

However, the present experiments demonstrate the difficulty of a complete quantitative characterization of the final state of spreading: if $S$ is large, the final pancake is so thin that roughness becomes a leading effect. Small $S$ values have to be achieved to increase the equilibrium thickness of the film, but then, it becomes highly sensitive to small $S$ variations.

A more quantitative analysis has been performed with the profiles of figure 5 [15], and we just recall here the results. The analysis of the precursor film profile performed by de Gennes and Joanny $[2,3]$ assumes that the liquid is injected in the film at a 
velocity $U=\mathrm{d} R / \mathrm{d} t$. The precursor film profile is then obtained by writing that the incoming liquid flows under the additional action of Laplace and disjoining pressure gradients in the film arising from curvature and thickness variations. Assuming a Poiseuille type flow, with a vanishing velocity at the solid wall leads to a differential equation for the film profile (for Van der Waals interactions) :

$$
\frac{3 \eta U}{z^{2}}=\frac{\mathrm{d}}{\mathrm{d} x}\left(-\gamma \frac{\mathrm{d}^{2} z}{\mathrm{~d} x^{2}}-\frac{A}{6 \pi z^{3}}\right)
$$

( $A$ is the effective Hamaker constant of the problem).

If the curvature term is negligible (small thickness region) equation (2) has a simple solution

$$
z \sim \frac{a}{\left(x-x_{0}\right) w}
$$

where $a=(A / 6 \pi \gamma)^{1 / 2}, w=\eta U / \gamma$, and $x_{0}$ is an integration constant to be determined by a suitable connection to the full profile. The dotted line on the insert of figure 5 is the best least square fit of the tail of the profile 1 on such a function. The agreement appears satisfactory. However, adjustment of the full profile on the calculated form (taking curvature terms into account), discussed in detail in reference [15], appears to be impossible. The films develop more rapidly than predicted, and the effect appears much more pronounced for the higher molecular weight. One may think that this is due to a trivial polydispersity effect, but the polydispersity of the high molecular weight sample is much smaller than that of the low molecular weight sample (see Tab. I), and the observed discrepancy can hardly be attributed to the polydispersity alone.

We rather think that this discrepancy between predicted and observed behaviour reveals specific polymeric effects. An indication that specific polymeric effects exist is the transient bump observed for $z \sim R_{\mathrm{G}}$ (Fig. 6). These effects are not classical non newtonian behaviour: for the two profiles of figure 5 , the average advancing velocities are respectively $2 \times 10^{-7}$ and $3 \times 10^{-10} \mathrm{~cm} / \mathrm{s}$, corresponding to a maximum shear rate, in the thinner part of the films of order respectively of 2 to $3 \times 10^{-3} \mathrm{~s}^{-1}$, well below the expected threshold for the appearance of non newtonian behaviour of the corresponding polymers. Specific polymeric effects on the spreading behaviour have been predicted by de Gennes and Brochard [18], noticing that the flow boundary condition at the solid wall for an entangled polymer should rather be a finite velocity [19]. Such slipping at the wall should modify both the macroscopic drop profile (appearance of a foot) and the precursor film profile which, far from the macroscopic drop, should rather decay like $z \sim \frac{1}{\sqrt{x-x_{0}}}$.
In our experiments we have not seen any of these behaviours. Many complications could occur and prevent slipping at the wall, as for example an anomalous structuration of the liquid close to the wall due to the high disjoining pressure [20], but this seems to be hardly compatible with the very thin final thickness we observe. Moreover, these structurations would rather slow down the process and we observe an acceleration. An alternative explanation could be that the long range forces are not correctly modelized by simple Van der Waals forces, and/or that the viscosity in such a thin polymer film is not the bulk liquid viscosity. We are presently trying to test these hypothesis.

A funny result, and apparently simple one, is illustrated in figure 7 where the dotted line is the best least square fit of the experimental profile of the tiny drop on a Gaussian curve. The agreement is quite good and it was impossible to obtain similar results with a Lorentzian, which is the shape predicted by de Gennes in this case [21]. We have, at the present time, no explanation for the profile or the spreading kinetics of such small drops.

\section{Conclusion.}

We have presented a series of experiments developed to try to understand the spreading behaviour of small drops of a non volatile liquid deposited on smooth solid surfaces. Several complementary techniques have been used to try to completely characterize the drop profile at all length scales. We have paid a special attention to the thin precursor film which develops all around the drop, first because precursor film studies on well controlled system are not numerous, and second, because this precursor film is of fundamental importance to understand the role of long range forces on spreading.

Our present results are in qualitative agreement with the theoretical predictions of Joanny and de Gennes: the spreading drop behaves in a very different way depending on the length scale. For thicknesses larger than the range of the interactions, we are in the macroscopic drop whose spreading kinetic is independent of the surface. For thicknesses smaller than the interaction range, one enters the precursor film which is deeply influenced both by the state of the macroscopic drop and by the spreading parameter. At fixed spreading parameter, the precursor film is more developed when the apparent contact angle of the macroscopic drop is smaller. For small spreading parameter values, the precursor film is highly sensitive to local inhomogeneities of the surface, and may be used, along with ellipsocontrast observation techniques, as a characterization tool of surface imperfections. When the precursor film thickness becomes small, roughness drives the spreading. 
Quantitative discrepancies remain between the observed and predicted precursor film profiles which may reveal either an incorrect description of the long range interactions by simple Van der Waals terms or an anomalous viscosity for liquid thicknesses smaller than the chain radius of gyration. Complementary experiments and analysis are presently underway to test these points.

\section{References}

[1] Teletzke, G. F., Davis, H. T., Scriven, L. E., J. Colloid Interface Sci. to be published.

[2] De Gennes, P. G., Rev. Mod. Phys. 57 (1985) 827.

[3] JoAnny, J. F., PHD thesis Université Paris VI (1985) ;

Joanny, J. F., J. Theor. Appl. Mech. 0750-7240 (1986) 249.

[4] Deryagin, B., Zh. Fiz. Khim. 14 (1940) 137.

[5] Marmur, Adv. Colloid Interface Sci. 19 (1983) 75.

[6] For a review on the dynamic properties of entangled polymers, see for example GrAESSLEY, W. W., Faraday Symp. Chem. Soc., 18 (1983) 315.

[7] VIG, J. R., Vac. Technol. A 3 (1985) 1027.

[8] Gun, J., SAGIV, J., J. Colloid Interface Sci. 112 (1986) 457.

[9] Shafrin, E. G., Zisman, W. A., J. Colloid Sci. 7 (1952) 166.

[10] Allain, C., Ausserré, D., Rondelez, F., J. Colloid Interface Sci. 107 (1985) 5.

[11] Born, M., Wolf, E., Principle of Optics (Pergamon, Oxford) 1980.
[12] Ausserre, D., Picard, A. M., Léger, L., Phys. Rev. Lett. 57 (1986) 2671.

[13] Erman, M., Theeten, J. B., J. Appl. Phys. 60 (1986) 859 ;

ERMAN, M., PHD thesis, Université Paris VI (1986).

[14] Azzam, R. M. A., Bashara, N. M., Ellipsometry and Polarized Light (North Holland Publishing Company, Amsterdam) 1977.

[15] Léger, L., Erman, M., Guinet-Picard, A. M., Ausserre, D., Strazielle, C., submitted to Phys. Rev. Lett.

[16] Pomerantz, M., Segmüller, A., Netzer, L., SagIV, J., Thin Solid Films 132 (1985) 153.

[17] Tanner, L., J. Phys. D 12 (1979) 1473.

[18] Brochard, F., De Gennes, P. G., J. Phys. Lett. France 45 (1984) L597.

[19] De Gennes, P. G., C.R. Hebd. Séan. Acad. Sci. Paris B 298 (1979) 219.

[20] Horn, R. G., IsRaelachvili, J. N., submitted for publication in Macromolecules.

[21] De Gennes, P. G., C. R. Hebd. Séan. Acad. Sci. Paris série II 298 (1984) 475. 